\title{
Outflow Vessel in the Plane of Main Vortex of Large Cerebral Aneurysms: A Study of Hemodynamic Analyses
}

\author{
Hao Li1, Tangming Peng1, Jing $\mathrm{Wu}^{2}$, Changren Huang1, Yong Jiang1, Ligang Chen ${ }^{*}$ \\ ${ }^{1}$ Department of Neurosurgery, The Affiliated Hospital of Luzhou Medical College, \\ Luzhou, China \\ ${ }^{2}$ Department of Interventional Neuroradiology, Beijing Tiantan Hospital, Capital Medical University, \\ Beijing, China \\ Email: lihao scmedical@126.com, ${ }^{*}$ pxtxmx@126.com
}

Received 9 March 2015; accepted 12 June 2015; published 15 June 2015

Copyright (C) 2015 by authors and Scientific Research Publishing Inc.

This work is licensed under the Creative Commons Attribution International License (CC BY). http://creativecommons.org/licenses/by/4.0/

(c) () Open Access

\begin{abstract}
Purpose: This study was designed to quantify and characterize the variations of hemodynamic parameters for those large cerebral aneurysms with outflow vessel in the plane of main vortex. Materials and Methods: A total of 19 consecutive patients with large cerebral aneurysms were constructed with the data of digital subtraction angiography. Those large cerebral aneurysms with outflow vessel in the plane of main vortex were included. Blood flow was hypothesized to be laminar and incompressible and blood Newtonian fluid. Computational fluid dynamics ICEM and Fluent software were used to simulate the computational hemodynamics of large cerebral aneurysms. Results: Hemodynamics parameters result of computational fluid dynamics showed that the velocity in the aneurysm neck, impact fields and the origin area of outflow vessels was obvious higher than that in the aneurysm sac and aneurysm dome. Wall shear stress was obvious higher in aneurysm neck, impact fields and the origin area of outflow vessels than that in the aneurysm sac and aneurysm dome. Conclusions: The location of outflow vessel played an impact on the level of blood flow within aneurysm sac for those large cerebral aneurysms with outflow vessel in the plane of main vortex.
\end{abstract}

\section{Keywords}

Aneurysm, Hemodynamic, Outflow Vessel, Vortex

\footnotetext{
${ }^{*}$ Corresponding author.
} 


\section{Introduction}

Hemodynamic status is regarded as one of the most significant factors responsible for the growth, development and rupture of cerebral aneurysms [1]. Hemodynamic variables such as flowpattern, wall shear stress (WSS) and wall shear stress gradient (WSSG) have been hypothesized to be the causes of aneurysms [2] [3]. The previous published reports also showed that the rupture risk of large aneurysm was higher than that of small aneurysms [4] [5]. Therefore, it is very important and essential for clinicians to master the hemodynamic status of aneurysms to evaluate and predict rupture risk, which is helpful in preoperative planning and preemptive treatments of cerebral aneurysms. In this study, using computational fluid dynamics (CFD) and angiographic image analysis, our objectives are to quantify and characterize the variations of hemodynamic parameters for those large cerebral aneurysms with outflow vessel in the plane of main vortex.

\section{Material and Methods}

\subsection{Patient Data}

Both study subject of Ethics Committee of our hospital is approved and written informed consent to participate in the study is provided. All the clinical records and radiologic images were reviewed and evaluated. A total of 19 consecutive patients, from February 2011 to May 2013, with large cerebral aneurysms with outflow vessel in the plane of main vortex, admitted to the Interventional Neuroradiology Department and imaged by digital subtraction angiography (DSA) are included in this study. Of those patients, age ranges from 32 to 63 years, with the average age of 49 years. Of which seven patients are males, accounting for $36.8 \%$ of our people.

\subsection{Image Data}

All catheter angiograms were performed by standard transdermal catheterizations of the cerebral arteries. Digital subtraction angiography imaging was performed on an uniplane unit (Philips Healthcare, Best, the Netherlands). Three-dimensional reconstruction of angiographic was performed in all 19 patients, with a result that the wideneck was larger than $15 \mathrm{~mm}$. In the data of the 19 large cerebral aneurysms, all located in posterior communicating artery. Of which, eight aneurysms were located in left posterior communicating artery, and the remained in right posterior communicating artery.

\subsection{Numerical Simulation of Hardware and Software}

The main hardware for professional work Station is Intel XeonE5 processors, 16 cores, memory 64 GB, hard disk 2000 GB. The main software is Fluent 13.0 (ANSYS company, USA), ANSYS CFD ICEM 13.0 (ANSYS company, USA).

\subsection{Hemodynamics Modeling}

Hypothesised that Newtonian fluid was viscous fluid with laminar flow, the controlled equations of which is non-steady incompressible Navier-Stokes equations [6]. Reynolds number of intravascular was ranging from 600 to 700 , the density of fluid was $1.05 \times 10^{3} \mathrm{~kg} / \mathrm{m}^{3}$, the viscosity coefficient of fluid was $0.0035 \mathrm{~Pa} \cdot \mathrm{s}$. It's also hypothesised that vessel wall and aneurysm wall were rigid walls, vessel inlet conditions was set as pulsating flow obtained using Doppler ultrasound and vessel export conditions was set as free flow. Methods of numerical simulation was proceeded as follows, the angiography images and records of cerebral aneurysms were obtained using the DSA post-processing workstation, the process, including triming and smoothing the small branches of vessel wall was furthermore performed. Therefore, the three-dimensional construction model of aneurysm and parent artery was established [5] [7]. The three-dimensional construction model of aneurysm was firstly meshed in ANSYS CFD ICEM 13.0 (ANSYS company, USA), and hemodynamic numerical simulation was then performed in Fluent 13.0 (ANSYS company, USA).

\subsection{Data Collection}

The computed artery and aneurysm flow fields were visualized by using a variety of techniques, including the following: 1) isovelocity surfaces to depict the aneurysm inflow stream; 2) velocity magnitudes on cut planes to 
depict the inflow jets and velocity profiles at the multiple dots; and 3) shaded surfaces to depict the distribution of wall shear stress magnitudes. The multiple dots contained the impact of aneurysm flow, aneurysm neck, aneurysm body, aneurysm tip, and aneurysm parent artery (including both sides of $0.5 \mathrm{~cm}$ from aneurysm neck). Spss 17.0 was used to data analysis.

\section{Results}

Both the relative location of outflow vessels of large intracranial aneurysms and the incident direction of blood flow affect the blood flow levels in aneurysm sac. After the blood flow entering into the aneurysm sac along the aneurysm wall, and then the most obvious vortex was firstly formed. In the present study, the plane with the most obvious vortex was defined as the main vortex. Those large cerebral aneurysms with outflow vessel in the plane of main vortex were included.

\section{Streamlines and Velocity}

After inlet flow across the aneurysm neck from the parent aneurysm artery, the inlet flow, impacting on the lateral wall of aneurysm, formed an impact field, and then flowed along the aneurysm wall to the outlet of aneurysm. Due to outlet vessels located in the plane of main vortex, the shunt effect was obvious in the outflow vessels, only remained a weak and slow vortex in the aneurysm sac. Hemodynamics parameters result of CFD showed that the velocity in the aneurysm neck, impact fields and the origin area of outflow vessels was obvious higher than that in the aneurysm sac and aneurysm dome (Table 1 and Figures 1(a)-(f)).

\section{Wall Shear Stress}

Wall shear stress distribution in aneurysm sac was similar as the blood velocity distribution, that is, wall shear stress was obvious higher in aneurysm neck, impact fields and the origin area of outflow vessels. On the contrary, wall shear stress was lower in the fields of aneurysm sac and aneurysm dome (Table 1 and Figures 1(a)-(f)).

\section{Discussion}

It is considered that the incidence of unruptured cerebral aneurysms was as high as $4.1 \%$ in population [7]-[9]. In addition, the diagnosed rate of unruptured cerebral aneurysms was raised apparently due to the development of noninvasive cerebral vascular imaging techniques, such as computed tomography angiography and magnetic resonance angiography. Cerebral aneurysm is a life-threatening disease due to the high rupture risk, thromboembolic, or compression of adjacent tissue. In recent published reports, CFD was used to study the cerebral circulation in an early stage. However, few had attempted to simulate hemodynamics, especially in outflow vessel in the plane of main vortex of large cerebral aneurysms [10] [11].

The present study was designed to identify the hemodynamic parameters, such as streamlines, velocity and WSS for large cerebral aneurysms with outflow vessel in the plane of main vortex. In the present study, intraaneurysmal hemodynamic forces, particularly streamlines, velocity and WSS, which were the tangential force produced by blood moving along the endothelial surface. There were more and more evidences showed that it played an important key in the evolution of aneurysmal disease [12]-[15]. These hemodynamic parameters were impacted on endothelial cell and leaded to changes the cell shape and structure. In the previous published reports, data showed that high WSS was demonstrated to initiate aneurysm formation, whereas low WSS leads to spatial disorganization of endothelial cells and a disregulation of antioxidant and antiinflammatory mediators resulting in arterial wall remodeling [5] [14] [16]. These factors could also be major contributors to aneurysm growth, due to slow recirculating flow, which leaded to large regions of low WSS could be observed in most aneurysms.

In the previous data of recent years, many studies on hemodynamics of cerebral aneurysms showed that large cerebral aneurysms possessed higher risk of rupture than small aneurysms, other than that the large cerebral aneurysms may be related to the hemodynamics [8] [17]. In addition, the hemodynamic features were different between large cerebral aneurysms and small cerebral aneurysms. In our series of large cerebral aneurysms with outflow vessel in the plane of main vortex, only triming and smoothing the small branches of vessel wall was furthermore performed, only leading to mimic effect on the CFD results.

From the view of fluid mechanics, geometry form between cerebral aneurysm and parent artery played a de, 
Table 1. Hemodynamics parameters in different area of aneurysms with outflow vessel in the plane of main vortex.

\begin{tabular}{ccc}
\hline & Velocity $(\mathrm{m} / \mathrm{s})$ & Wall shear stress $(\mathrm{Pa})$ \\
\hline Aneurysm dome & $0.009 \pm 0.004$ & $0.007 \pm 0.003$ \\
Aneurysm sac & $0.139 \pm 0.013$ & $0.672 \pm 0.273$ \\
Parent artery & $0.745 \pm 0.044$ & $8.315 \pm 1.115$ \\
Aneurysm dome/parent artery & $0.012 \pm 0.005$ & $0.003 \pm 0.001$ \\
Aneurysm sac/parent artery & $0.186 \pm 0.019$ & $0.081 \pm 0.029$
\end{tabular}

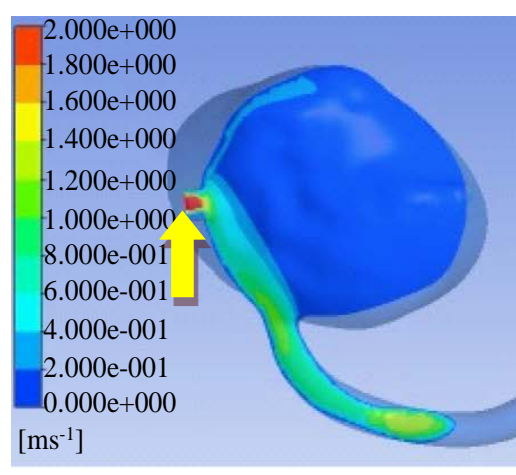

(a)

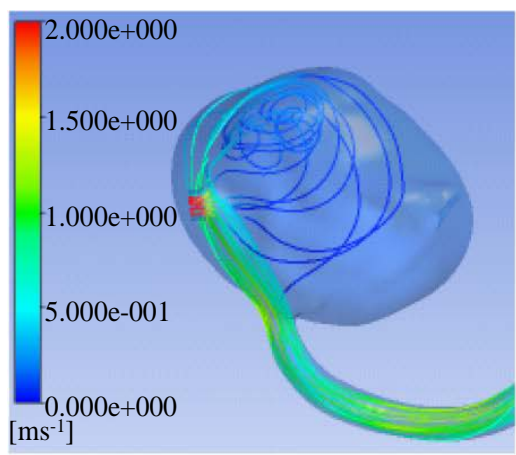

(d)

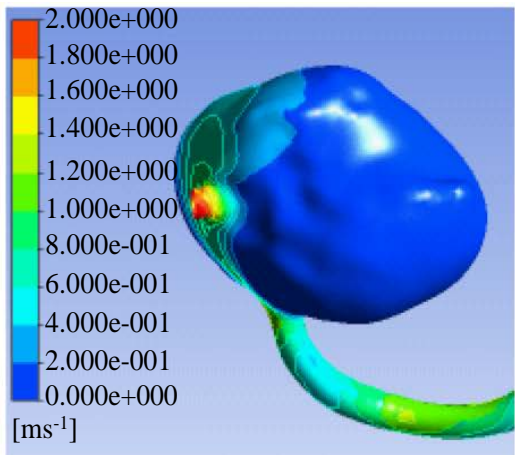

(b)

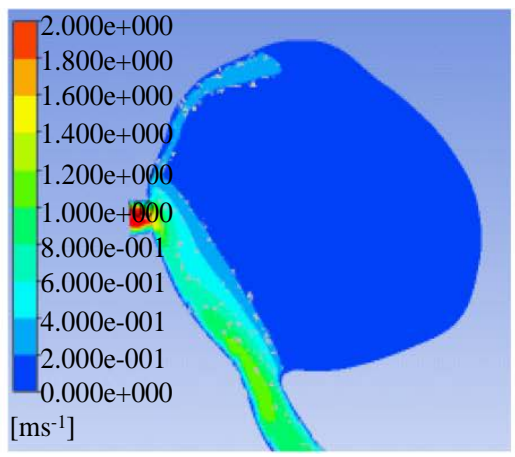

(e)

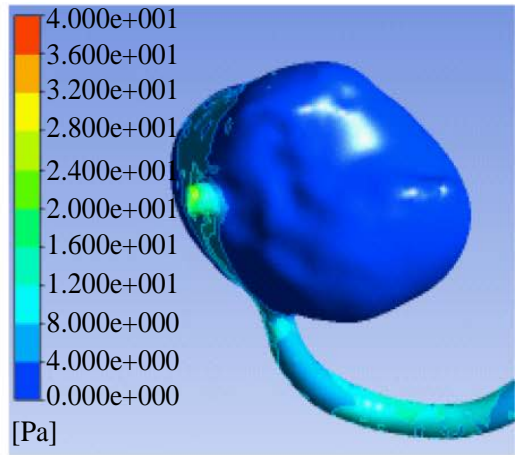

(c)

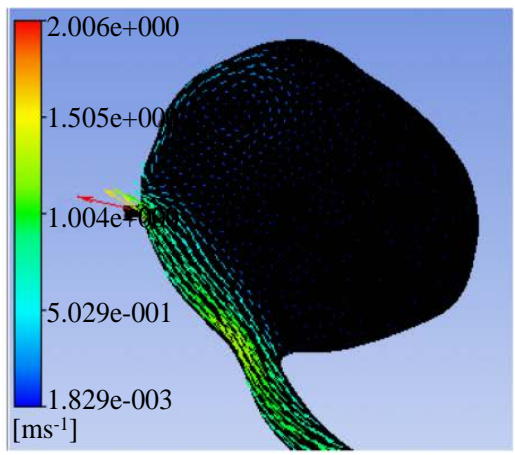

(f)

Figure 1. (a)-(f) Showed aneurysm outflow vessel in the plane of main vortex. (a) The Main vortex (red arrow) and outflow vessels (yellow arrow); (b) Flow Velocity in the lateral wall of aneurysm; (c) Wall shear stress; (d) Streamlines; (e) Flow velocity of the main vortex; (f) Streamlines direction of the main vortex. In compared velocity with wall shear stress obviously, velocity ruler was set as $0-2 \mathrm{~m} / \mathrm{s}$, wall shear stress was set as $0-40 \mathrm{~Pa}$.

cisive impact on the flow field in aneurysm sac. In previous reports, data showed that aneurysm aspection ration vascular curvature of parent artery also affected the blood flow level [13] [18] [19]. In the present study, the result of CFD also demonstrated that the location of outflow vessels played an impact on the blood flow. In compared with the small cerebral aneurysms, it's more complex for large cerebral aneurysms between dome and outflow vessel, and large cerebral aneurysms were more difficult to define as side aneurysm or tip aneurysm [20]. In the present study, low velocity and wall shear stress in aneurysm sac and aneurysm dome were found in those large cerebral aneurysms with outflow vessel in the plane of main vortex, which may be one of the factors for those aneurysms in predicting rupture.

There were several limitations in the present study. Firstly, the samples were small, and need more samples to identify the location of outflow's effect on hemodynamics. Secondly, the location of aneurysm, aneurysm neck size and other aneurysm morphology factors may also play a key role in blood flow. Therefore, these bias described above should be taken into consideration in followed study. 


\section{Conclusion}

The location of outflow vessel played an impact on the level of blood flow within aneurysm sac for those large cerebral aneurysms with outflow vessel in the plane of main vortex.

\section{Article Supported}

No.

\section{References}

[1] Tateshima, S., Tanishita, K., Hakata, Y., et al. (2009) Alteration of Intraaneurysmal Hemodynamics by Placement of a Self-Expandable Stent. Laboratory investigation. Journal of Neurosurgery, 111, 22-27. http://dx.doi.org/10.3171/2009.2.JNS081324

[2] Tremmel, M., Xiang, J., Natarajan, S.K., et al. (2010) Alteration of Intra-Aneurysmal Hemodynamics for Flow Diversion Using Enterprise and Vision Stents. World Neurosurgery, 74, 306-315. http://dx.doi.org/10.1016/j.wneu.2010.05.008

[3] Hakimi, M., Knez, P., Lippert, M., et al. (2012) Altered In-Stent Hemodynamics May Cause Erroneous Upgrading of Moderate Carotid Artery Restenosis When Evaluated by Duplex Ultrasound. Journal of Vascular Surgery, 56, 14031408. http://dx.doi.org/10.1016/j.jvs.2012.03.035

[4] Boussel, L., Rayz, V., McCulloch, C., et al. (2008) Aneurysm Growth Occurs at Region of Low Wall Shear Stress: Patient-Specific Correlation of Hemodynamics and Growth in a Longitudinal Study. Stroke, 39, 2997-3002. http://dx.doi.org/10.1161/STROKEAHA.108.521617

[5] Pereira, V.M., Brina, O., Gonzalez, A.M., et al. (2013) Biology and Hemodynamics of Aneurismal Vasculopathies. European Journal of Radiology, 82, 1606-1617. http://dx.doi.org/10.1016/j.ejrad.2012.12.012

[6] Meng, H., Tutino, V.M., Xiang, J., et al. (2014) High WSS or Low WSS? Complex Interactions of Hemodynamics with Intracranial Aneurysm Initiation, Growth, and Rupture: Toward a Unifying Hypothesis. AJNR American Journal of Neuroradiology, 35, 1254-1262. http://dx.doi.org/10.3174/ajnr.A3558

[7] Huang, Q., Xu, J., Cheng, J., et al. (2013) Hemodynamic Changes by Flow Diverters in Rabbit Aneurysm Models: A Computational Fluid Dynamic Study Based on Micro-Computed Tomography Reconstruction. Stroke, 44, 1936-1941. http://dx.doi.org/10.1161/STROKEAHA.113.001202

[8] Wu, C., Xu, B.N., Sun, Z.H., et al. (2012) Different Treatment Modalities of Fusiform Basilar Trunk Aneurysm: Study on Computational Hemodynamics. Chinese Medical Journal, 125, 97-101.

[9] Takao, H., Murayama, Y., Otsuka, S., et al. (2012) Hemodynamic Differences between Unruptured and Ruptured Intracranial Aneurysms during Observation. Stroke, 43, 1436-1439. http://dx.doi.org/10.1161/STROKEAHA.111.640995

[10] Zhang, Y., Mu, S., Chen, J., et al. (2011) Hemodynamic Analysis of Intracranial Aneurysms with Daughter Blebs. European Neurology, 66, 359-367. http://dx.doi.org/10.1159/000332814

[11] Chien, A., Castro, M.A., Tateshima, S., Sayre, J., Cebral, J., et al. (2009) Quantitative Hemodynamic Analysis of Brain Aneurysms at Different Locations. American Journal of Neuroradiology, 30, 1507-1512. http://dx.doi.org/10.3174/ajnr.A1600

[12] Liu, J., Xiang, J.P., Zhang, Y., Wang, Y., Li, H.Y., et al. (2014) Morphologic and Hemodynamic Analysis of Paraclinoid Aneurysms: Ruptured versus Unruptured. Journal of Neurointerventional Surgery, 6, 658-663. http://dx.doi.org/10.1136/neurintsurg-2013-010946

[13] Duan, G.L., Lv, N., Yin, J.H., Xu, J.Y., Hong, B., et al. (2014) Morphological and Hemodynamic Analysis of Posterior Communicating Artery Aneurysms Prone to Rupture: A Matched Case-Control Study. Journal of Neurointerventional Surgery, 12, 456-460. http://dx.doi.org/10.1136/neurintsurg-2014-011450

[14] Yan, L., Zhu, Y.-Q., Li, M.-H., Tan, H.-Q. and Cheng, Y.-S. (2013) Geometric, Hemodynamic, and Pathological Study of a Distal Internal Carotid Artery Aneurysm Model in Dogs. Stroke, 44, 2926-2929. http://dx.doi.org/10.1161/STROKEAHA.113.002290

[15] Metaxa, E., Tremmel, M., Natarajan, S.K., Xiang, J.P., Paluch, R.A., et al. (2010) Characterization of Critical Hemodynamics Contributing to Aneurysmal Remodeling at the Basilar Terminus in a Rabbit Model. Stroke, 41, 1774-1782. http://dx.doi.org/10.1161/STROKEAHA.110.585992

[16] Xu, J.Y., Yu, Y., Wu, X., Wu, Y.F., Jiang, C., et al. (2013) Morphological and Hemodynamic Analysis of Mirror Posterior Communicating Artery Aneurysms. PloS ONE, 8, e55413. http://dx.doi.org/10.1371/journal.pone.0055413

[17] Kono, K., Fujimoto, T., Shintani, A. and Tomoaki, T. (2012) Hemodynamic Characteristics at the Rupture Site of Cerebral Aneurysms: A Case Study. Neurosurgery, 71, E1202-E1208. http://dx.doi.org/10.1227/NEU.0b013e31826f7ede 
[18] Backes, D., Vergouwen, M.D., Velthuis, B.K., van der Schaaf, I.C., Bor, A.S.E., et al. (2014) Difference in Aneurysm Characteristics between Ruptured and Unruptured Aneurysms in Patients with Multiple Intracranial Aneurysms. Stroke, 45, 1299-1303. http://dx.doi.org/10.1161/STROKEAHA.113.004421

[19] Larrabide, I., Aguilar, M.L., Morales, H.G., Geers, A.J., Kulcsár, Z., et al. (2013) Intra-Aneurysmal Pressure and Flow Changes Induced by Flow Diverters: Relation to Aneurysm Size and Shape. American Journal of Neuroradiology, 34, 816-822. http://dx.doi.org/10.3174/ajnr.A3288

[20] Xiang, J.P., Natarajan, S.K., Tremmel, M., Ma, D., Mocco, J., et al. (2011) Hemodynamic-Morphologic Discriminants for Intracranial Aneurysm Rupture. Stroke, 42, 144-152. 